Bundesgesundheitsbl 2022 $65: 86-95$ https://doi.org/10.1007/s00103-021-03457-z Eingegangen: 1. September 2021

Angenommen: 3. November 2021

Online publiziert: 8. Dezember 2021

(c) Der/die Autor(en) 2021

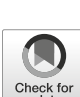

Maren Eggers ${ }^{1,2,3} \cdot$ Anna Baumann $^{4} \cdot$ Nils Lilienthal $^{4} \cdot$ Eike Steinmann $^{5} \cdot$ Jochen Steinmann ${ }^{6} \cdot$ Nils-Olaf Hübner ${ }^{7} \cdot$ Holger F. Rabenau $^{8} \cdot$ Viola Weinheimer $^{9}$. Ingeborg Schwebke ${ }^{1,2}$

${ }^{1}$ Kommission für Virusdesinfektion, Deutsche Vereinigung zur Bekämpfung der Viruskrankheiten (DVV) e. V., Geschäftsstelle Kiel, Kiel, Deutschland

${ }^{2}$ Gesellschaft für Virologie (GfV) e. V., Geschäftsstelle Heidelberg, Heidelberg, Deutschland

${ }^{3}$ Labor Prof. G. Enders MVZ GbR, Stuttgart, Deutschland

${ }^{4}$ Bundesinstitut für Arzneimittel und Medizinprodukte (BfArM), Bonn, Deutschland

${ }^{5}$ Abteilung für Molekulare \& Medizinische Virologie, Ruhr-Universität Bochum, Bochum, Deutschland

${ }^{6}$ Dr. Brill + Partner GmbH Institut für Hygiene und Mikrobiologie, Bremen, Deutschland

${ }^{7}$ Institut für Hygiene und Umweltmedizin, Universitätsmedizin Greifswald, Greifswald, Deutschland

${ }^{8}$ Institut für Medizinische Virologie, Universitätsklinikum Frankfurt am Main, Frankfurt am Main, Deutschland

${ }^{9}$ Bundesanstalt für Arbeitsschutz und Arbeitsmedizin (BAuA), Dortmund, Deutschland

\title{
Desinfektionsmittel in der COVID-19-Pandemie: eine Herausforderung
}

die Nachfrage nach Desinfektionsmitteln auch in Bereichen, in denen diese bisher nicht oder kaum eingesetzt worden waren, stark an. Dies betraf zum einen Verbraucher aus Sorge, sich im öffentlichen Umfeld mit SARS-CoV-2 $\mathrm{zu}$ infizieren - obwohl in verschiedenen Stellungnahmen darauf hingewiesen wurde, dass Händewaschen in der Regel ausreicht und eine Flächendesinfektion im Haushalt nicht erforderlich ist, so in den Stellungnahmen der Weltgesundheitsorganisation (WHO), des Robert Koch-Instituts (RKI), der Bundeszentrale für gesundheitliche Aufklärung (BZgA), der Desinfektionsmittelkommission der Deutschen Vereinigung zur Bekämpfung der Viruskrankheiten (DVV) und der Gesellschaft für Virologie (GfV; [2-4]).

Auch in vielen öffentlichen Bereichen, wie z.B. Supermärkten, Restaurants und Bahnhöfen, hoffte man durch Flächenund Händedesinfektion die Pandemie eindämmen zu können. Vor allem nach dem ersten Lockdown setzten die örtlichen Behörden in Deutschland auf Hände- und Flächendesinfektion im öffentlichen Bereich. Allein für die Schulträger in Nordrhein-Westfalen wurden 20.0001 Desinfektionsmittel zum Ab- ruf gestellt [5]. Durch diese zusätzliche Nachfrage nach Desinfektionsmitteln im Inland und bereits seit Anfang Januar durch das Ausland (z. B. China) kam es in Deutschland zur starken Verknappung etablierter Produkte.

Eine Steigerung der Produktion war nur eingeschränkt möglich, da auch Verpackungen und Rohstoffe in der erforderlichen Qualität extrem knapp wurden. Die bisher verfügbaren Desinfektionsmittel, vor allem für die Händedesinfektion, waren zu diesem Zeitpunkt kaum noch erhältlich. Deshalb wurde intensiv nach Lösungen gesucht, um den Mangel möglichst schnell zu beseitigen und die Desinfektion vor allem im medizinischen Bereich im gewohnten Qualitäts- und Sicherheitsstandard durchführen zu können. Dazu war eine Kommunikationsstrategie notwendig, die den Anwender informieren, aber nicht verunsichern sollte. Währenddessen tauchten im Handel immer mehr neue und ungewöhnliche Produkte auf (• Abb. 1). In Deutschland wurden in der Folge die Empfehlungen auf anerkannte Verfahren zur Desinfektion beschränkt.

Inwieweit die ergriffenen Maßnahmen zur Desinfektion ihrem Ziel gerecht 


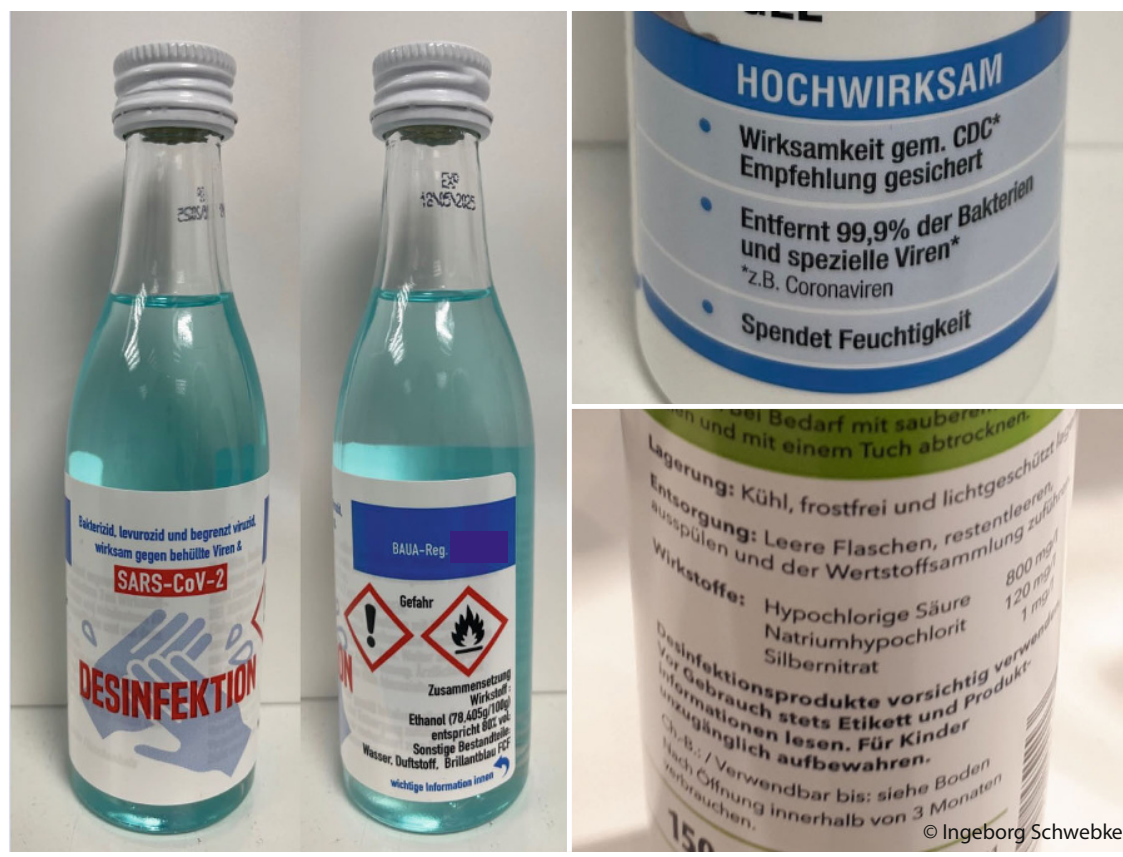

Abb. $1 \Delta$ Beispiele für ungewöhnliche Verpackungen, Deklarationen und Wirkstoffe von Desinfektionsmitteln, die während der COVID-19-Pandemie in den Handel kamen. (Fotos mit freundlicher Genehmigung $\odot$ Ingeborg Schwebke)

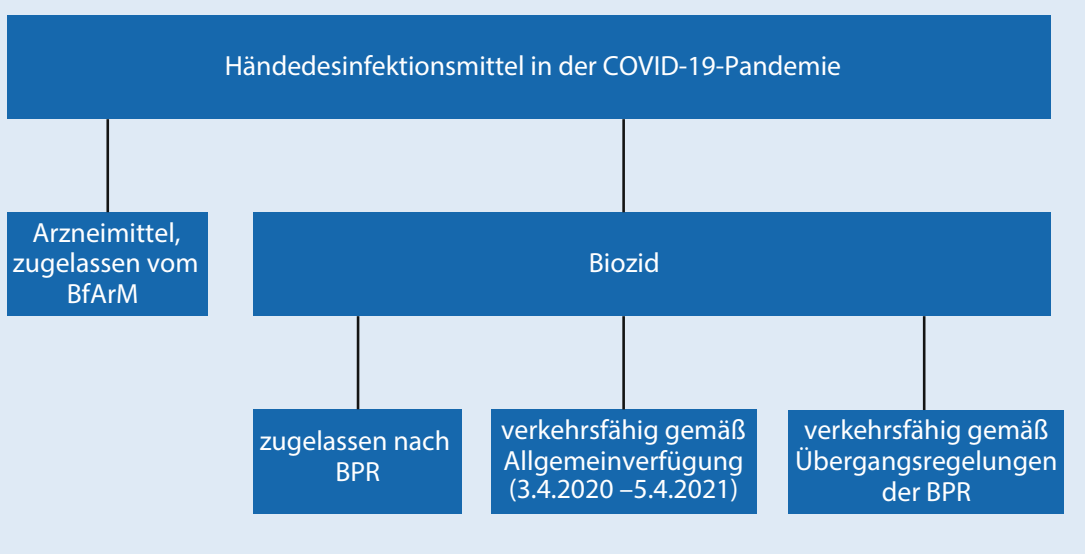

Abb. $2 \Delta$ Grundlagen der Verkehrsfähigkeit von Händedesinfektionsmitteln in Deutschland während der COVID-19-Pandemie. BfArM Bundesinstitut für Arzneimittel und Medizinprodukte, BPR BiozidVerordnung (Biocidal Products Regulation)

wurden bzw. welche Bedeutung sie speziell bei SARS-CoV-2 haben, lässt sich nach fast 2 Jahren Pandemie auf der Basis von Erfahrungen und wissenschaftlichen Erkenntnissen genauer einschätzen. Der vorliegende Beitrag beschreibt zunächst die Anforderungen an chemische Hände- und Flächendesinfektionsmittel und die rechtlichen Grundlagen für ihre Verkehrsfähigkeit. Im Folgenden werden die auf dieser Basis ergriffenen Maßnahmen und die speziellen Fragestellungen bei der Organisation der Desinfektions- mittelversorgung dargestellt. Dabei wird auch auf die Besonderheiten der Übergangsregelungen im Biozidrecht eingegangen. Des Weiteren wird die Bedeutung der Flächendesinfektion bei SARSCoV-2 erörtert. Abschließend werden die während der Pandemie gewonnenen Erkenntnisse bei der Organisation gestellt und Lehren gezogen, die dazu beitragen sollen, bei zukünftigen Pandemien bezüglich der Desinfektion besser vorbereitet zu sein. der Desinfektionsmittelversorgung dar-

\section{Anforderungen an chemische Desinfektionsmittel}

Desinfektionsmittel müssen für den ausgelobten Wirkbereich (z.B. Bakterien, Hefen, Sporen, Viren) wirksam und für die jeweilige Anwendung weitgehend unbedenklich für Mensch und Umwelt sein. Der Nachweis der Wirksamkeit für den entsprechenden Wirkbereich muss durch standardisierte Prüfmethoden erbracht werden. Dazu existieren europäische Normen, deren Anwendung in der übergeordneten Norm DIN EN 14885 [6] geregelt ist. In Deutschland werden zum Teil darüber hinausgehende nationale Prüfvorschriften des Verbundes für Angewandte Hygiene e. V. (VAH), der DVV e. V. und GfV e. V. sowie des RKI angewendet. In jedem Fall sind orientierende Suspensionsversuche und praxisnahe Tests mit den für das jeweilige Wirkspektrum vorgegebenen Testorganismen durchzuführen. Nähere Erläuterungen zum erforderlichen Wirkspektrum und dem Nachweis der Wirksamkeit mit standardisierten Methoden für Händedesinfektionsmittel wurden im Epidemiologischen Bulletin 2020 veröffentlicht [7].

Aufgrund der biochemischen Struktur und der Erfahrungen mit SARSCoV-1 war bekannt, dass zur Desinfektion alkoholische Lösungen gut geeignet sind. Wie SARS-CoV-1 gehört SARSCoV-2 zu den behüllten Viren, sodass zu seiner Inaktivierung der Wirkbereich „begrenzt viruzid“ ausreichend ist. Allgemeine Anforderungen an den Nachweis der Viruswirksamkeit hat der Arbeitskreis Viruzidie beim RKI formuliert [8].

Im medizinischen Bereich muss aber immer auch mit dem Vorhandensein weiterer Krankheitserreger gerechnet werden, sodass als Grundvoraussetzung bei Desinfektionsmitteln, neben einer Wirksamkeit gegen behüllte Viren (begrenzt viruzid), auch die Wirksamkeit gegen Bakterien und Hefen (Bakterizidie und Levurozidie) gegeben sein muss. Diese Anforderung gilt auch für den öffentlichen Bereich, wie z. B. Schulen, Feuerwehr oder Polizei, sowie für den privaten Bereich, wenn hier aus medizinischen Gründen eine Desinfektion erforderlich ist. 
Desinfektionsmittel sollten jedoch nicht nur wirksam, sondern auch sicher in der Anwendung sein, was auch für die zu Pandemiebeginn dringend benötigten Produktalternativen galt.

\section{Rechtliche Grundlagen für die Verkehrsfähigkeit chemischer Desinfektionsmittel}

Händedesinfektionsmittel können rechtlich Biozidprodukte der Produktart 1 (PT 1, Produkte für die menschliche Hygiene) sein oder aufgrund von $\mathrm{Be}$ standsschutz Arzneimittel (• Abb. 2) sein. Flächendesinfektionsmittel gehören in der Regel zu den Biozidprodukten und hier zur Produktart 2 (PT 2). Unter Pandemiebedingungen waren vorübergehende juristische Regelungen für beide Rechtskreise, Biozidprodukte und Arzneimittel, erforderlich.

\section{Händedesinfektionsmittel als Arzneimittel oder Biozidprodukt}

Neue Händedesinfektionsmittel werden als Biozidprodukte zugelassen, es sei denn, die beantragten Indikationen beinhalten zusätzlich zur hygienischen und chirurgischen Händedesinfektion auch die Verhütung von Krankheiten (z.B. Wundantiseptik; [9]). Die Zulassungsanforderungen für Händedesinfektionsmittel zum Beleg der Wirksamkeit ergeben sich allgemein aus der angestrebten Indikation - hygienische oder chirurgische Händedesinfektion - und dem beantragten Wirkspektrum.

Unterschiede in den Zulassungsanforderungen zwischen Händedesinfektionsmitteln als Arzneimittel und als Biozidprodukt wurden zuletzt ausführlich im Epidemiologischen Bulletin 17/2021 diskutiert [10]. Die Belege zum Wirksamkeitsnachweis stimmen jedoch weitestgehend überein.

Während der Pandemie wurde jedoch nicht nur die Neuzulassung von Händedesinfektionsmitteln wieder interessant, sondern auch die erweiterte Anwendung bereits zugelassener Händedesinfektionsmittel in der Verantwortung des Bundesinstituts für Arzneimittel und Medizinprodukte (BfArM). So ergaben sich in 6 Fällen Zulassungsänderungen

Bundesgesundheitsbl 2022 ·65:86-95 https://doi.org/10.1007/s00103-021-03457-z (c) Der/die Autor(en) 2021

M. Eggers · A. Baumann - N. Lilienthal · E. Steinmann - J. Steinmann - N.-O. Hübner · H. F. Rabenau · V. Weinheimer · I. Schwebke

\section{Desinfektionsmittel in der COVID-19-Pandemie: eine Herausforderung}

\section{Zusammenfassung}

Durch die COVID-19-Pandemie haben Desinfektionsmaßnahmen auch in Deutschland an Bedeutung gewonnen. Der erhöhte Bedarf an Desinfektionsmitteln zu Beginn der Pandemie erforderte es, vorübergehende rechtliche Regelungen zu treffen, um einerseits ausreichend Mittel für die notwendige Desinfektion im medizinischen Bereich und andererseits für den zusätzlichen Bedarf in der Bevölkerung zur Verfügung zu haben. Dazu wurden vom Bundesinstitut für Arzneimittel und Medizinprodukte (BfArM) und der Bundesanstalt für Arbeitsschutz und Arbeitsmedizin (BAuA) Allgemeinverfügungen erlassen, die in diesem Beitrag näher erläutert werden. Im Vordergrund stehen dabei die Maßnahmen für die hygienische Händedesinfektion. Aber auch weitere Anwendungen wie die Flächendesinfektion im Zusammenhang mit pandemischen Atemwegserkrankungen werden erörtert. Die Erfahrungen bei der Sicherstellung der Versorgung mit wirksamen und in der Anwendung sicheren Desinfektionsmitteln sollten für die Vorbereitung weiterer Pandemien genutzt werden.

Schlüsselwörter

Coronavirus SARS-CoV-2 - Desinfektion . Allgemeinverfügungen · Arzneimittel · Biozidprodukte

\section{Disinfectants during the COVID-19 pandemic: a challenge}

\section{Abstract}

Disinfection measures have become more important as a result of the COVID-19 pandemic in Germany. The increased need for disinfectants at the beginning of the pandemic required temporary legal regulations in order to provide a sufficient quantity of products for the necessary disinfection in the medical sector on the one hand and for the additional demand in the population on the other. For this purpose, the Federal Institute for Drugs and Medical Devices (BfArM) and the Federal Institute for Occupational Safety and Health (BAuA) issued a general ruling, which is explained in more detail in this article. The focus was on measures for hygienic hand disinfection. However, other applications such as surface disinfection in relation to pandemic respiratory diseases are also addressed. The experience gained in ensuring the supply of disinfectants that are effective and safe to use should be used to prepare for further pandemics.

\section{Keywords}

Coronavirus SARS-CoV-2 - Disinfection . General ruling $\cdot$ Medicinal products $\cdot$ Biocidal products zur Erweiterung des Wirkspektrums auf „begrenzt viruzid“ bzw. „begrenzt viruzid PLUS“, um die Wirksamkeit gegen behüllte Viren, die SARS-CoV-2 einschließen, bzw. zusätzlich gegen die unbehüllten Adeno-, Rota- und Noroviren, ausloben zu können.

\section{Flächendesinfektionsmittel als Biozidprodukt}

Flächendesinfektionsmittel werden von den jeweiligen nationalen Zulassungsbehörden (in Deutschland: Bundesanstalt für Arbeitsschutz und Arbeitsmedi- zin [BAuA]) bzw. der Europäischen Chemikalienagentur (ECHA) auf der Basis der Anforderungen der Biozid-Verordnung (BPR; [11]) und zugehöriger Dokumente als Biozidprodukte zugelassen, Informationen hierzu siehe Website der BAuA [12].

\section{Ausnahmezulassungen nach Biozid-Verordnung (BPR)}

Der Artikel 55 der BPR [11] regelt Ausnahmen des Zulassungsverfahrens. Das heißt, „eine zuständige Behörde“ kann „befristet für eine Dauer von höchstens 


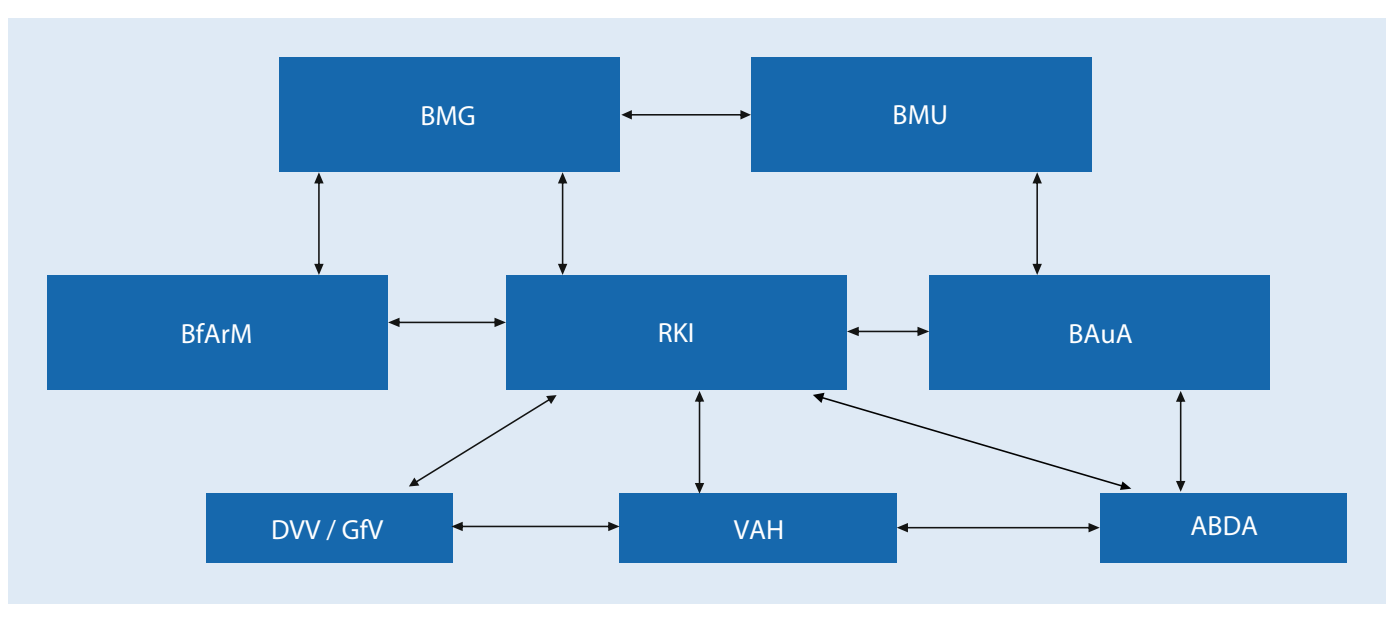

Abb. $3 \Delta$ Zusammenarbeit von Ministerien, Oberen Bundesbehörden und Verbänden bei der Erstellung der Allgemeinverfügungen, um den erhöhten Bedarf an Desinfektionsmitteln zu Beginn der Pandemie zu regeln. $A B D A$ Bundesvereinigung Deutscher Apothekerverbände e. V., BAuA Bundesanstalt für Arbeitsschutz und Arbeitsmedizin, BfArM Bundesinstitut für Arzneimittel und Medizinprodukte, $B M G$ Bundesministerium für Gesundheit, $B M U$ Bundesministerium für Umwelt, Naturschutz und nukleare Sicherheit, DVV Deutsche Vereinigung zur Bekämpfung der Viruskrankheiten e. V., GfV Gesellschaft für Virologie e. V. (DVV/GfV Kommission Virusdesinfektion), RKI Robert Koch-Institut, VAH Verbund für Angewandte Hygiene e.V.

180 Tagen die Bereitstellung eines Biozidprodukts auf dem Markt oder die Verwendung eines Biozidprodukts, das nicht die in dieser Verordnung niedergelegten Voraussetzungen für die Erteilung einer Zulassung erfüllt, für eine beschränkte und kontrollierte Verwendung unter der Aufsicht der zuständigen Behörde gestatten, wenn dies aufgrund einer Gefahr für die öffentliche Gesundheit, die Tiergesundheit oder für die Umwelt notwendig ist, die mit anderen Mitteln nicht eingedämmt werden kann“. SARS-CoV-2 stellt zwar keine besonders hohen Anforderungen an Desinfektionsmittel, aber die üblichen Produkte waren schnell nicht mehr in ausreichender Menge verfügbar. Deshalb trat dieser Artikel im humanmedizinischen Bereich erstmals in Kraft, damit auch nicht regulär zugelassene Produkte sicher angewendet werden konnten.

Einzelheiten für das Vorgehen nach Artikel 55 BPR sind für Deutschland in $\$ 12$ Chemikaliengesetz (ChemG; [13]) festgelegt. Die BAuA als reguläre Zulassungsbehörde verfügte über jahrelange Erfahrung in Zulassungsverfahren; hier jedoch mussten schnell Produkte für die „Ausnahmezulassung" gefunden werden, die einerseits wirksam und andererseits auch sicher in der Anwendung sind. In enger Zusammenarbeit mit dem RKI und den beiden zuständigen $\mathrm{Mi}$ nisterien - Ministerium für Umwelt,
Naturschutz und nukleare Sicherheit (BMU) sowie dem Bundesministerium für Gesundheit (BMG) - entstanden Allgemeinverfügungen für die hygienische Händedesinfektion und die Flächendesinfektion ([14, 15]; • Abb. 3).

\section{Wege aus der Produktknapp- heit - Allgemeinverfügungen und Standardzulassung}

Um der Dringlichkeit des pandemiegeschuldet erhöhten Bedarfs an Händedesinfektionsmitteln Rechnung zu tragen, wurden zeitlich befristete Abweichungen von den allgemeinen Standards der Zulassung für alkoholhaltige Arzneimittel und Biozidprodukte zur hygienischen Händedesinfektion erlaubt. Hierzu erließen das BfArM und die BAuA im März 2020 Allgemeinverfügungen, um die Verfügbarkeit von Händedesinfektionsmitteln unter Beibehaltung eines hohen Mindeststandards aufrechtzuerhalten $[14,16]$.

\section{Allgemeinverfügung des BfArM für Händedesinfektionsmittel}

Die Allgemeinverfügung des BfArM [16] erlaubte beispielsweise - vorbehaltlich der Gewährleistung gleicher Unbedenklichkeit und Wirksamkeit gegen Viren Freiheiten bei der Wahl des Wirkstofflieferanten, der Packmittel und deren Far- ben sowie die Substitution nicht wirksamkeitsrelevanter Hilfsstoffe. Zusätzlich wurde die Spezifikation zur Sporenfreiheit in Arzneimitteln ausgesetzt, um die Freigabe zu beschleunigen. Durch diese Allgemeinverfügung konnte bei mindestens 2 Firmen die Lieferfähigkeit essenzieller Produkte zur Händedesinfektion aufrechterhalten werden. Die Allgemeinverfügung des BfArM bezog sich jedoch ausdrücklich nur auf Desinfektionsmittel zur hygienischen Händedesinfektion. Für die chirurgische Händedesinfektion wurden keine vorrübergehenden Abweichungen ermöglicht, um den hohen Standards der Krankenhaushygiene und dem Schutz der Patienten vor postoperativen Infektionen gerecht $\mathrm{zu}$ werden.

\section{Allgemeinverfügung der BAuA für Händedesinfektionsmittel}

Die Allgemeinverfügung der BAuA [14] beinhaltete die Herstellung und Verwendung 2-propanolhaltiger und ethanolhaltiger Händedesinfektionsmittel nach vorgegebenen Rezepturen.

Der Versorgungsengpass bei Desinfektionsmitteln führte auch schnell $\mathrm{zu}$ der Frage einer möglichen Eigenherstellung und den damit verbundenen Fragen: Welche Rezepturen sind geeignet und was ist bei der Herstellung zu beachten? Erste Überlegungen führten $\mathrm{zu}$ den WHO-Formulierungen [17], die vor- 
rangig für Länder gedacht sind, die keine eigene Produktion besitzen und die die relativ teuren Produkte der westlichen Welt nicht erwerben können. Diese Fragen wurden bereits im März 2020 über die Veröffentlichung der Desinfektionsmittelkommission der DVV e. V. und GfV e. V. [2] parallel zur ersten Allgemeinverfügung der BAuA adressiert.

Die wichtigsten Wirkstoffe der etablierten Händedesinfektionsmittel sind Ethanol, Isopropanol (2-Propanol) und 1-Propanol. Nachdem konfektionierte Produkte mit diesen Wirkstoffen nicht mehr zur Verfügung standen, wurden für die Allgemeinverfügung die beiden WHO-Formulierungen I und II [17] auf der Basis von Ethanol bzw. Isopropanol herangezogen, da zu ihrer Wirksamkeit - auch gegen Viren - eine Vielzahl von Publikationen vorlag [18-20]. In nachfolgenden wissenschaftlichen Untersuchungen bestätigte sich ihre Wirksamkeit auch gegenüber dem behüllten SARS-CoV-2 [21].

Neben den in Deutschland vor der Pandemie nicht verwendeten WHO-Formulierungen existieren im Arzneimittelbereich die Standardzulassungen verschiedener alkoholischer Lösungen, für deren Wirksamkeit auch gegen behüllte Viren ebenfalls wissenschaftliche Untersuchungen vorlagen und die in die Desinfektionsmittelliste des RKI aufgenommen waren [22-25]. Sie haben z.T. zusätzlich den Anwendungsbereich der Hautdesinfektion, für den ebenfalls die Mittel knapp wurden.

Über die korrekte Herstellung der Lösungen herrschten allerdings vor allem bei in der Desinfektionsmittelproduktion unerfahrenen Firmen viele Unklarheiten. Lösungen z. B. mit einem vorgegebenen Ethanolgehalt herzustellen ist keineswegs trivial, da substanztypische Eigenschaften wie die Volumenkontraktion bei Verdünnung und die Dichte zu beachten sind. Mit Unterstützung der Bundesvereinigung Deutscher Apothekerverbände e.V. (ABDA) gelang es, eine nachvollziehbare Herstellungsanweisung zu erstellen, die dann in der endgültigen Fassung der Allgemeinverfügung veröffentlicht wurde [14]. Eine solche detaillierte Vorschrift wurde notwendig, da aus Kapazitätsgründen nun nicht nur die erfah- renen Hersteller, sondern auch weitere Firmen der pharmazeutischen und chemischen Industrie sowie juristische Personen des öffentlichen Rechts die Möglichkeit erhielten, die Lösungen gemäß Allgemeinverfügung herzustellen.

Hintergründe zur Wirksamkeit und Unbedenklichkeit der verschiedenen Rezepturen wurden detailliert in einem Artikel des RKI zusammen mit der DVV und GfV und weiteren Experten zum Tag der Händehygiene 2020 dargestellt [7]. Der VAH veröffentlichte zeitgleich eine weitere Mitteilung [26], die beinhaltete, dass nur bei Ethanol-Wasser-Gemischen ab $80 \%(\mathrm{v} / \mathrm{v})^{1}$ und 2-Propanol-WasserGemischen ab $75 \%(\mathrm{v} / \mathrm{v})$ auch von einer ausreichenden bakteriziden Wirksamkeit nach 0,5 min Einwirkzeit ausgegangen werden kann. Für die Anwendung von Hautantiseptika empfahl die VAHDesinfektionsmittelkommission vorzugsweise die alkoholischen Lösungen der Standardzulassung zu nutzen, da bei sachgerechter Herstellung gemäß den entsprechenden Monografien der Standardzulassung des BfArM auch eine Sporenfreiheit der Lösungen gegeben wäre. Bei Produkten, die als Biozide oder gemäß der von der BAuA erlassenen Allgemeinverfügung vermarktet wurden, muss dies nicht der Fall sein.

\section{Standardzulassungen für alkoholische Lösungen}

Standardzulassungen stellen bestimmte Fertigarzneimittel gemäß $₫ 36$ Arzneimittelgesetz (AMG) von der Zulassungspflicht frei. Voraussetzung hierfür ist, dass keine Gefährdung von Menschen und Tieren zu befürchten ist. Sie basieren auf Monografien, die das BMG in Kraft setzt. Sie werden im Bundesanzeiger veröffentlicht und dürfen nur den jeweiligen Alkohol (auch mit Butan-2on vergällt) und Wasser enthalten. Ihre Herstellung unter Normalbedingungen bedarf der Anzeige beim BfArM und der zuständigen Landesbehörde.

Diese Ausnahme von der Zulassungspflicht über die Standardzulassung wurde in den letzten Jahren teilweise

\footnotetext{
1 (v/v) Konzentrationsangabe in Volumenprozent.
}

kritisch gesehen, da keine tiefergehende Prüfung der Anträge auf Standardzulassung erfolgte und diese Regelung eine nationale Besonderheit darstellt, die im harmonisierten europäischen Arzneimittelrecht nicht vorkommt. Sie erlaubte aber während der Pandemie Apotheken, pharmazeutischen und chemischen Unternehmen sowie juristischen Personen des öffentlichen Rechts relativ unproblematisch Händedesinfektionsmittel herzustellen. So gingen nach Jahren ohne neue Anzeigen ab Pandemiebeginn Mitte März 2020 insgesamt 35 neue Anzeigen von Standardzulassungen alkoholischer Lösungen beim BfArM ein. Die Hürden für die Herstellung wurden durch die Allgemeinverfügung nochmals herabgesetzt.

Eine der Lehren aus der Pandemie sollte daher sein, über den Fortbestand der Standardzulassung für diese Alkohole nachzudenken, um in der Zukunft flexibler auf Lieferengpässe reagieren zu können.

Mit zunehmender Verbesserung der Versorgungsstruktur und Verfügbarkeit von Desinfektionsmitteln liefen die Allgemeinverfügung des BfArM [16] zum 30.09.2020 und die Allgemeinverfügungen der BAuA [14] zum 05.04.2021 aus.

\section{Mehr Freiheiten bei der pharmazeutischen Qualität von Händedesinfektionsmitteln}

Speziell bei Händedesinfektionsmitteln ist die Qualität der Rohstoffe besonders wichtig, da hier ein Produkt regelmäßig auf lebendes Gewebe - die menschliche Haut - aufgebracht wird. Bereits die erste Version der Allgemeinverfügung der BAuA berücksichtigte diesen Aspekt, indem die Qualität der Mittel so definiert wurde, dass weder CMR (kanzerogene, mutagene, reproduktionstoxische) noch sensibilisierende Bestandteile über einem zulässigen Grenzwert $(\leq 0,1 \%)$ enthalten sein durften.

Im Gegensatz zu regulär zugelassenen Händedesinfektionsmitteln, für die die Art der Verpackung vorgeschrieben ist, wurde durch die Allgemeinverfügungen auch die Verpackung in riesigen Gebinden - bis 10001 - möglich. Doch wie kann aus solchen Gebinden eine Abfül- 
lung in handliche Gefäße unter hygienischen Bedingungen erfolgen? Da es im Biozidrecht keine Vorgaben zur mikrobiologischen Reinheit gibt, blieb das eine rechtlich ungelöste Frage, die geeignete Antworten durch die Anwender und Überwachungsbehörden forderte.

Außerdem waren die wichtigsten Wirkstoffe für Händedesinfektionsmittel - Ethanol und Isopropanol - nicht in ausreichender Menge in der erforderlichen pharmazeutischen Qualität verfügbar. Um auch weitere Quellen für alkoholische Wirkstoffe nutzen $\mathrm{zu}$ können, wurde der Artikel 95 der BPR [11] ausgesetzt. Dieser Artikel verweist auf eine Liste der Wirkstoffhersteller, die die Dossiers für das jeweilige Wirkstoffgenehmigungsverfahren erstellt haben und nutzen dürfen. Daher zeigte sich auch unter Pandemiebedingungen die essenzielle Bedeutung des Wirkstoffes Ethanol, da hierfür notfalls weitere Quellen verfügbar wären und Ethanol zur Inaktivierung von Viren eine große Bedeutung hat [27].

Es ergab sich allerdings ein Problem: So wollten z.B. Spirituosen- und Bierhersteller Ethanol aus ihrer eigenen Herstellung zur Verfügung stellen. Ein nicht näher charakterisierter Rohethanol kann aber nicht ohne Weiteres für die Anwendung auf der Haut freigegeben werden, da u.a. Fuselstoffe die Haut reizen können. Aber nicht nur die Reinheit, sondern auch der Ethanolgehalt solcher Rohfraktionen war nicht in jedem Fall geeignet.

Verunreinigungen von Produkten mit Methanol, die in den USA zu umfangreichen Warnhinweisen und Rückrufen geführt hatten $[28,29]$, wurden auch in Deutschland festgestellt. So mussten 2 Einzelhandelsketten ein Produkt (Handgel) zurückrufen, dass 65,3 g beziehungsweise $68,7 \mathrm{~g}$ Methanol pro $100 \mathrm{~g}$ Produkt enthielt $^{2}$ [30]. Das Produkt stammte von einem Großhändler für alkoholische Ge-

2 www.produktwarnung.eu: „Ein Teil der Produkte enthält eine hohe Menge an Methanol (65,3 g bzw. $68,7 \mathrm{~g}$ pro $100 \mathrm{~g}$ ), das eine toxische Wirkung hat. Der Hersteller verfügt nicht über die Erlaubnis, die Produkte in Verkehr zu bringen und hat die Artikel nicht mit den notwendigen kindergesicherten Verschlüssen versehen. Gesundheitsschädliche Auswirkungen können nicht ausgeschlossen werden, insbesondere tränke, Tabakwaren, Kosmetik und Feinkost, der keine Genehmigung zum Verkauf des Handgels hatte.

\section{Allgemeinverfügung für Flächendesinfektionsmittel}

Neben der Allgemeinverfügung für Händedesinfektionsmittel, konnte vom 02.04.2020 bis 30.09.2020 eine Allgemeinverfügung der BAuA zum Inverkehrbringen von Flächendesinfektionsmitteln genutzt werden [15]. Sie enthielt $80 \%(\mathrm{v} / \mathrm{v})$ Ethanol-, 0,5\% (w/w $\left.\mathrm{w}^{3}\right)$ Natriumhypochlorit- und 2,5\% (w/w) Chloramin-T-Lösung mit entsprechenden Qualitätsvorgaben.

\section{Übergangsregelungen im Biozidrecht}

Neben diesen nun für den „Notfall“ per Allgemeinverfügung zugelassenen Produkten gibt es aufgrund der Übergangsregelungen für Biozidprodukte mit sogenannten Altwirkstoffen in Deutschland auch viele weitere Produkte mit anderen Wirkstoffen, die derzeit noch ohne $\mathrm{Zu}$ lassung verkehrsfähig sind. Diese werden vom Inverkehrbringer lediglich elektronisch bei der BAuA gemäß der Meldeverordnung [31] registriert. Eine behördliche Prüfung hinsichtlich ihrer Wirksamkeit, Unbedenklichkeit und Qualität ist für derartige Meldungen gesetzlich nicht vorgeschrieben. Man erkennt sie an der Registriernummer mit der Struktur „Nxxxxx“.

Die gemeldeten Biozidprodukte mit Altwirkstoffen, also Produkte, die ausschließlich Wirkstoffe enthalten, die entsprechend der Review-Verordnung (Verordnung (EU) Nr. 1062/2014; [32]) bewertet wurden bzw. bewertet werden, können in Deutschland bis zur (Nicht-)Genehmigung der enthaltenen Wirkstoffe ohne Zulassung vermarktet und verwendet werden. Bis spätestens zum Zeitpunkt der Genehmigung des Wirkstoffs muss jedoch ein vollständiger

bei Verschlucken, Hautkontakt oder Einatmen des Inhalts. Von einem Gebrauch wird daher abgeraten".

${ }^{3}(\mathrm{w} / \mathrm{w})$ Konzentrationsangabe in Massenprozent.
Antrag auf Zulassung gestellt werden, um die Verkehrsfähigkeit zu erhalten. Enthält ein Produkt mehrere Altwirkstoffe, gilt als Frist der Zeitpunkt des letzten $\mathrm{zu}$ genehmigenden Wirkstoffs (vorausgesetzt, es kam nicht zu einer Nichtgenehmigung eines der Wirkstoffe).

\section{Spielräume der Übergangs- regelungen für neue oder (wieder-)entdeckte Produkte}

Einige „neue“ Produkte bzw. bereits auf dem Markt befindliche Produkte mit Aktivchlor als Wirkstoff wurden während der Pandemie (wieder-)entdeckt und teils aggressiv beworben (• Abb. 1).

Die oben genannten Übergangsregelungen wurden von Herstellern, deren Produkte bei Desinfektionsmaßnahmen im medizinischen Bereich in der Regel nicht zur ersten Wahl gehörten, verstärkt genutzt. Aufgrund des Mangels an geeigneten alkoholischen Rohstoffen im Frühjahr 2020 sprangen Hersteller von Chlorprodukten in diese Lücke und bewarben ihre Produkte für die Bekämpfung der Pandemie. Diese Produkte enthielten vorrangig Aktivchlor, das aus Natriumhypochlorit, hypochloriger Säure oder Salzlösungen hergestellt ist. Die Mehrzahl der Produkte war durch Übergangsregelungen verkehrsfähig und noch nicht bewertet.

Für Produkte zur Händedesinfektion (d.h. PT 1) mit dem Wirkstoff Natriumhypochlorit hätte bereits zum 01.01.2019 ein Zulassungsantrag gestellt werden müssen. Einige Firmen fanden allerdings Wege, den erforderlichen Zulassungsantrag zu umgehen. Dazu setzten sie z.B. Ethanol in minimaler Menge $\mathrm{zu}$, da dieser Wirkstoff noch nicht genehmigt ist, oder meldeten das entsprechende Produkt zusätzlich für eine Produktart an, für die das Aktivchlor noch nicht genehmigt war, wie z. B. PT 11 - Schutzmittel für Flüssigkeiten in Kühl- und Verfahrenssystemen. Damit war es möglich, solche Aktivchlor abspaltenden Produkte ohne Zulassung, d.h. ohne eine Bewertung, sogar für die Händedesinfektion zu vermarkten.

Aufgrund des allgemeinen Desinfektionsmittelmangels wurden diese Pro- 
dukte nun auch in größerem Umfang genutzt. Dadurch offenbarten sich aber auch ihre Schwachpunkte wie relativ lange Einwirkzeiten oder die häufig geringe Haltbarkeit infolge der Instabilität der Wirkstoffe. Überwachungsbehörden berichteten dem RKI zudem mehrfach von stark schwankenden Wirkstoffgehalten. Somit erfordert die Anwendung solcher Produkte eine regelmäßige Überprüfung des Wirkstoffgehalts. Zur Information der Anwender hatte die Desinfektionsmittelkommission des VAH zwei diesbezügliche Mitteilungen veröffentlicht $[33,34]$. Aufgrund der fehlenden Erfahrung mit häufigen Anwendungen wird darin von der Verwendung chlorhaltiger Produkte für die Händedesinfektion abgeraten, solange diese nicht zugelassen sind, und eine Listung wird von der Zulassung abhängig gemacht.

Aus Sicht der Arzneimittelzulassung sind diese Produkte prinzipiell zur Händedesinfektion geeignet. Allerdings ist von einer allgemeinen Verwendung chlorhaltiger Produkte zur Händedesinfektion abzuraten, da Natriumhypochloritlösungen in der Regel pH-Werte deutlich im alkalischen Bereich aufweisen, während der $\mathrm{pH}$-Wert der Haut im schwach sauren Bereich liegt. Hautirritationen können bei einer wiederholten Verwendung grundsätzlich nicht ausgeschlossen werden. Für die wiederholte hygienische Händedesinfektion werden alkoholbasierte Händedesinfektionsmittel empfohlen [35]. Dennoch wird auch hier eine Intensivierung der Hautpflegemaßnahmen nahegelegt. Aus den oben genannten Gründen des Hautschutzes wird nur die Verwendung ausschließlich für die Händedesinfektion zugelassener Produkte angeraten.

Die Zuordnung des richtigen Wirkstoffes bei Produkten, die Aktivchlor entwickeln, ist kompliziert. Deshalb hat die BAuA im Helpdesk hierzu eine Information (FAQ Nr. 0598) veröffentlicht [36]. Inzwischen ist die Bewertung weiterer Aktivchlor abspaltender Wirkstoffe abgeschlossen und sie wurden als ,genehmigte" Wirkstoffe in die Unionsliste [37] für PT 1 und PT 2 (z. B. als Flächendesinfektionsmittel) aufgenommen. Damit ist auch ein Ende der Nutzung von Übergangsregelungen vorgegeben. Im Rahmen der
Bewertung im Zulassungsverfahren wird sich zeigen, unter welchen Gegebenheiten Aktivchlor abspaltende Verbindungen sicher zur Desinfektion eingesetzt werden können.

\section{Flächendesinfektion bei SARS-CoV-2}

Bei zahlreichen Virusinfektionen erfolgt die Übertragung humanpathogener Viren nicht nur über Tröpfchen oder aerogen, sondern auch über kontaminierte Flächen. Dabei ist im Allgemeinen die Stabilität behüllter, humanpathogener Viren wie SARS-CoV-2 durch den Lipidanteil in ihrer Membran geringer als die unbehüllter Viren (z.B. Noroviren). Wichtige Faktoren für die Stabilität der Viren in der Umwelt sind ferner mögliche Eiweiß- und Blutzusätze, die Temperatur, die Luftfeuchtigkeit und die Art bzw. das Material der Fläche. Nach der Übersicht von Kramer et al. liegt die Zeit bis zur Inaktivierung auf der Fläche bei behüllten Viren aus dem Bereich des Respirationstraktes und dem derblutübertragenen Viren (HIV, Hepatitisviren) im Bereich von wenigen Tagen, während für unbehüllte Viren, die überwiegend aus dem Gastrointestinaltrakt stammen, sogar Wochen und Monate angegeben werden [38, 39].

$\mathrm{Zu}$ Beginn und auch im weiteren Verlauf der COVID-19-Pandemie ist zurecht dann auch immer wieder hinterfragt worden, wo, wie und in welchem Umfang eine Kontamination von patientennahen Flächen durch Personen mit positivem SARS-CoV-2-Nachweis im Nasen-/Rachenabstrich erfolgen kann. Deshalb sind bereits zu Beginn der Pandemie Untersuchungen zum Nachweis und zur Stabilität von SARS-CoV-2 auf unterschiedlichen Flächen erfolgt [40, 41]. Viele der erhobenen Daten zur Stabilität auf der Fläche basieren jedoch nicht auf der Detektion von infektiösen Viruspartikeln, nachgewiesen in der Zellkultur, sondern von genetischem Material, welches in diesen Fällen mit der Polymerasekettenreaktion (PCR) detektiert wird. Eine Studie aus Wuhan zeigte beispielsweise, dass mit der PCR in Patientenzimmern das genetische Material bis zu 28 Tage nachgewiesen werden konnte [42]. Ein Virusnachweis in der Zellkultur ist hier nicht versucht worden. In einer anderen Studie wurde in $52,3 \%$ der Proben in einem Londoner Krankenhaus genetisches Material detektiert [43]. Ein direkter Nachweis infektiöser Viren gelang hier nicht. Eine Studie aus einem Krankenhaus in Singapur ergab ein unklares Bild der Viruskontamination mit negativen und positiven Befunden, wobei die Nachweise mit der PCR hauptsächlich im Badezimmer erfolgreich waren [44]. Diese und viele weitere Studien zeigen das Ausmaß einer möglichen Kontamination im medizinischen Bereich. Der Versuch einer Virusisolierung fehlt aber häufig bzw. zeigte ein negatives Ergebnis. Deshalb wurde in der Folge intensiv diskutiert, welche Bedeutung positive PCR-Befunde für die Notwendigkeit einer Flächendesinfektion im medizinischen Bereich überhaupt haben [45].

Ein wichtiges Hilfsmittel für die Bewertung PCR-positiver Proben könnte der sogenannte Ct(Cycle-Threshold)Wert sein, mit dem beschrieben wird, wie viele Zyklen in der PCR für die Generierung eines positiven Resultates erforderlich sind. Aber auch diese ergänzende Angabe ersetzt nicht den fehlenden Virusnachweis. Im nichtmedizinischen Bereich wird die durchgeführte Flächendesinfektion kritisch gesehen, wobei auch hier der fehlende Virusnachweis mit der Zellkultur als wichtiges Kriterium für die vorgenommene Einschätzung angesehen wird [46].

Daher weisen die US-amerikanischen Centers for Disease Control and Prevention (CDC) in einer im April 2021 aktualisierten Stellungnahme [47] darauf hin, dass durch Kontakt mit Oberflächen SARS-CoV-2 übertragen werden könnte. Die Übertragung über Oberflächen stellt - basierend auf den verfügbaren epidemiologischen Daten und Studien - jedoch nicht den Hauptübertragungsweg von SARS-CoV-2 dar und das Risiko wird als gering eingestuft. Klar ist, dassSARS-CoV-2 primär durch Aerosole oder Tröpfchen, die auf die Atemwegsschleimhäute gelangen, übertragen wird.

Daher wäre es in den meisten Situationen im nichtmedizinischen Bereich ausreichend, die Oberflächen mit Seife oder 
Reinigungsmittel $\mathrm{zu}$ reinigen und nicht zu desinfizieren.

In Situationen, in denen innerhalb der letzten $24 \mathrm{~h}$ ein Verdachtsfall oder ein bestätigter Fall von COVID-19 in Innenräumen aufgetreten ist, ist das Vorhandensein infektiöser Viren auf Oberflächen wahrscheinlicher. Die Desinfektion von häufig berührten Oberflächen sollte daher in Betracht gezogen werden [48].

Wie bei den Stabilitätsdaten auf der Fläche sind viele Rückschlüsse zur chemischen Inaktivierung des SARS-CoV-2 von anderen Mitgliedern der großen Coronavirusfamilie abgeleitet, wobei sicherlich von einem weitestgehend identischen Verhalten der einzelnen Familienmitglieder der Coronaviridae gegenüber chemischen Flächendesinfektionsmitteln ausgegangen werden kann.

Neuere gezielte Untersuchungen von Handelspräparaten zur Flächendesinfektion mit dem SARS-CoV-2 sind in Deutschland und Europa nur im Einzelfall erforderlich. In den vorhandenen Prüfnormen [6] werden in der Regel einzelne apathogene und möglichst gut zu vermehrende Vertreter mit hohen Titern in der Zellkultur als Prüfviren im quantitativen Suspensionsversuch und in praxisnahen Versuchen unter Belastung eingesetzt. Bekanntlich fungiert bei der Prüfung der Viruzide der chemischen Flächendesinfektionsmittel das Vacciniavirus als Prüfvirus für alle behüllte Viren (begrenzt viruzid) und erlaubt so auch eine sichere Aussage zur Wirksamkeit gegenüber dem SARSCoV-2 [49, 50].

Mit dieser Vorgehensweise bei der Prüfung von chemischen Desinfektionsmitteln können so geprüfte und zertifizierte Flächendesinfektionsmittel auch in Zeiten von COVID-19 zielgerecht zur Inaktivierung des SARSCoV-2 eingesetzt werden. Geprüfte Flächendesinfektionsmittel mit einem erweiterten Wirkbereich wie „begrenzt viruzid PLUS“ und „viruzid“ können ebenfalls erfolgreich bei der Inaktivierung des SARS-CoV-2 Verwendung finden.

\section{Lehren aus der Pandemie}

Die Erfahrungen aus knapp 2 Jahren COVID-19-Pandemie zeigen, dass mit Artikel 55 der BPR in Europa eine tragfähige rechtliche Vorgabe existiert, um im Bedarfsfall schnell zusätzliche Produkte zur Desinfektion verfügbar zu machen. Die größte Herausforderung bestand dabei darin sicherzustellen, dass trotzdem nur wirksame und verträgliche Produkte auf den Markt gelangen.

Hier zeigten sich die Grenzen der aktuellen Gesetzgebung: Weder das europäische noch das deutsche Biozidrecht enthielten Instrumente, um zu verhindern, dass unter Pandemiebedingungen ungeprüfte Produkte massenhaft vermarktet wurden. Nachbesserungen hinsichtlich der Verkehrsfähigkeit insbesondere für Händedesinfektionsmittel als Produkte, die unmittelbar auf der menschlichen Haut angewendet werden, erscheinen dringend geboten.

In jedem Fall müssen auch Produkte für eine „Notfallzulassung“ wirksam und sicher bzw. unbedenklich in der Anwendung sein. Die Prüfung der Wirksamkeit und Sicherheit muss schon vor einer Pandemie oder einer anderen „Gefahr für die öffentliche Gesundheit" erfolgen, da sie einen längeren Zeitraum in Anspruch nimmt. Somit ist es erforderlich, Produkte für ein breites Wirkspektrum vorzuhalten, um im Bedarfsfall schnell reagieren zu können, wozu über die $\mathrm{Zu}$ lassung der Biozidprodukte hinaus Desinfektionsmittellisten, wie z.B. die des RKI oder des VAH, beitragen können.

Eine weitere Lehre aus der Pandemie ist die Verbesserung der Vorsorge (Preparedness) zur Verhinderung von Mangelsituationen: Eine gravierende Desinfektionsmittelknappheit war vor der Pandemie nicht im Fokus der Vorbereitungsmaßnahmen. Zukünftig sollte daher die Sicherstellung der Versorgungssicherheit mit Desinfektionsmitteln und Antiseptika für den medizinischen Bereich in Zeiten stark erhöhter Nachfrage und des Mangels an Rohstoffen etc. Teil der Vorbereitung sein. Zusätzlich sollte über den Fortbestand der Standardzulassung für alkoholische Lösungen nachgedacht werden, um flexibler auf Lieferengpässe reagieren zu können.
Die Pandemie hat zudem deutlich gemacht, dass eine intensive Kooperation der mit Desinfektionsmitteln befassten Bundesbehörden und Ministerien für sachdienliche Lösungen unumgänglich ist. Es wäre wünschenswert, diese $\mathrm{Zu}$ sammenarbeit z. B. in einem Arbeitskreis dauerhaft zu regeln, um bei Produkten, die mit medizinischer Zweckbestimmung eingesetzt werden sollen, infektionshygienische und anwendungsrelevante Aspekte besser berücksichtigen zu können. Hierbei sollte zudem die in unabhängigen Expertengremien wie der DVV, dem VAH oder der Deutschen Gesellschaft für Krankenhaushygiene (DGKH) vorhandene Kompetenz eingebunden werden.

Derzeit ist die Versorgung mit Desinfektionsmitteln wieder gesichert und Ausweichprodukte sind entbehrlich. Das ist ein glücklicher Umstand und nicht selbstverständlich. Auch ist zu bedenken, dass es im Fall von SARS-CoV-2 als behülltem Virus recht unproblematisch war, geeignete Produkte zur sicheren Inaktivierung zu identifizieren. Bei stabileren Erregern und solchen, bei denen durch eine Händewaschung und Flächenreinigung keine ausreichende Wirkung zu erzielen ist, dürfte es schwieriger sein, geeignete Produkte zu identifizieren als auch bereitzustellen, da die Nachfrage nochmals wesentlich größer sein dürfte.

Daher ist die COVID-19-Pandemie auch als ein Test für die Krisenfestigkeit der Versorgung mit Desinfektionsmitteln zu sehen. Dabei sind die Stärken, aber auch die Defizite deutlich geworden. Der Blick in die Geschichte zeigt, dass Pandemien regelmäßige Ereignisse sind. $\mathrm{Ob}$ in der nächsten Pandemie eine sichere Versorgung mit Desinfektionsmitteln, Masken, persönlicher Schutzausrüstung etc. gegeben ist, wird dagegen maßgeblich davon abhängen, ob die Lehren aus dieser Pandemie berücksichtigt und die Defizite behoben werden.

Bei alledem sollten auch die Kommunikation und Transparenz zur Notwendigkeit und Sinnhaftigkeit von Desinfektionsmaßnahmen nicht unterschätzt werden. So ist die grundsätzliche Anwendung von Hände- und Flächendesinfektionsmitteln im Rahmen eines überwiegend respiratorisch übertrage- 
nen Erregers kritisch bzw. differenziert $\mathrm{zu}$ betrachten. Neben der sinnvollen und unzweifelhaften Anwendung von Desinfektionsmitteln im medizinischen Bereich hat die Desinfektionsmittelbereitstellung und Nutzung z.B. in Kaufhäusern, Restaurants oder im Privatbereich eher einen stark eingeschränkten medizinisch-biologischen Nutzen, während die psychologische Komponente dieser Maßnahme (im Sinne „jeder kann selbst etwas tun und ist nicht nur ausgeliefert") nicht unterschätzt werden darf. Nichtsdestotrotz sollte gerade für den Privatbereich bzw. überall dort, wo möglich, betont werden, dass man mit einfachem Händewaschen oftmals eine Übertragung effizient bekämpfen kann.

\section{Korrespondenzadresse}

\section{Dr. Maren Eggers}

Labor Prof. G. Enders MVZ GbR

Rosenbergstraße 85, 70193 Stuttgart,

Deutschland

eggers@labor-enders.de

\section{Einhaltung ethischer Richtlinien}

Interessenkonflikt. M. Eggers, A. Baumann, N. Lilienthal, E. Steinmann, J. Steinmann, N.-O. Hübner, H.F. Rabenau, V. Weinheimer und I. Schwebke geben an, dass kein Interessenkonflikt besteht.

Für diesen Beitrag wurden von den Autoren keine Studien an Menschen oder Tieren durchgeführt. Für die aufgeführten Studien gelten die jeweils dort angegebenen ethischen Richtlinien.

Open Access. Dieser Artikel wird unter der Creative Commons Namensnennung 4.0 International Lizenz veröffentlicht, welche die Nutzung, Vervielfältigung, Bearbeitung, Verbreitung und Wiedergabe in jeglichem Medium und Format erlaubt, sofern Sie den/die ursprünglichen Autor(en) und die Quelle ordnungsgemäß nennen, einen Link zur Creative Commons Lizenz beifügen und angeben, ob Änderungen vorgenommen wurden.

Die in diesem Artikel enthaltenen Bilder und sonstiges Drittmaterial unterliegen ebenfalls der genannten Creative Commons Lizenz, sofern sich aus der Abbildungslegende nichts anderes ergibt. Sofern das betreffende Material nicht unter der genannten Creative Commons Lizenz steht und die betreffende Handlung nicht nach gesetzlichen Vorschriften erlaubt ist, ist für die oben aufgeführten Weiterverwendungen des Materials die Einwilligung des jeweiligen Rechteinhabers einzuholen.

Weitere Details zur Lizenz entnehmen Sie bitte der Lizenzinformation auf http://creativecommons.org/ licenses/by/4.0/deed.de.

\section{Literatur}

1. https://www.worldometers.info/coronavirus/. Zugegriffen:30. Nov. 2021

2. Stellungnahme der Desinfektionsmittelkommission von DVV und GfV, Welches Desinfektionsmittel ist bei Verdacht auf bzw. Erkrankung mit dem neuen Coronavirus SARS-CoV-2 (COVID19, für Coronavirus disease 19) einzusetzen? https://g-f-v.org/welches-desinfektionsmittelist-bei-verdacht-auf-bzw-erkrankung-mit-demneuen-coronavirus-sars-cov-2-covid-19-fuercoronavirus-disease-19-einzusetzen/. Zugegriffen: 29.10.2021

3. https://www.euro.who.int/de/health-topics/ health-emergencies/coronavirus-covid-19/ news/news/2020/3/who-announces-covid-19outbreak-a-pandemic. Zugegriffen: 19. Okt. 2021

4. Hinweise zu Reinigung und Desinfektion von Oberflächen außerhalb von Gesundheitseinrichtungen im Zusammenhang mit der COVID-19Pandemie Stand: 3. Juli 2020. https://www.rki.de/ DE/Content/InfAZ/N/Neuartiges_Coronavirus/ Reinigung_Desinfektion.html. Zugegriffen 30 . Nov. 2021

5. https://www.land.nrw/en/node/23512.Zugegriffen: 19.0kt. 2021

6. DIN EN 14885 Chemische Desinfektionsmittel und Antiseptika - Anwendung Europäischer Normen für chemische Desinfektionsmittel und Antiseptika; Deutsche Fassung EN 14885:2018 Ausgabe 2019-10

7. Hübner NO, Eggers M, Schwebke I, Suchomel M (2020) Händedesinfektion unter den Bedingungen der SARS-CoV-2-Pandemie. Epid Bull 19:13-20. https://doi.org/10.25646/6861

8. Schwebke I, Eggers M, Gebel J et al (2017) Prüfung und Deklaration der Wirksamkeit von Desinfektionsmitteln gegen Viren zur Anwendung im human-medizinischen Bereich. Stellungnahme des Arbeitskreises Viruzidie beim Robert Koch-Institut (RKI), des Fachausschusses Virusdesinfektion der Deutschen Vereinigung zur Bekämpfung der Viruskrankheiten (DVV) e.V. und der Gesellschaft für Virologie (GfV) e.V. sowie der Desinfektionsmittelkommission des Verbundes für Angewandte Hygiene (VAH) e.V. Bundesgesundheitsblatt Gesundheitsforschung Gesundheitsschutz 60:353-363

9. OLG Urteil Köln. http://www.justiz.nrw. de/nrwe/ovgs/vg_koeln/j2011/7_K_5708_ 08urteil20111206.Html.Zugegriffen: 19.0kt. 2021

10. Lilienthal N,Baumann A, RespondekV,HübnerN-O, Schwebke I (2021) Quo vadis Händedesinfektionsmittel: Arzneimittel oder Biozidprodukt? Epid Bull 17:5-13. https://doi.org/10.25646/8389

11. Verordnung (EU) Nr. 528/2012 des Europäischen Parlaments und des Rates vom 22. Mai 2012 über die Bereitstellung auf dem Markt und die Verwendung von Biozidprodukten. 2012

12. BAuA Bundesstelle für Chemikalien https:// www.baua.de/DE/Themen/AnwendungssichereChemikalien-und-Produkte/Chemikalienrecht/ Chemikalienrecht_node.html. Zugegriffen: 19. Okt. 2021

13. Gesetz zum Schutz vor gefährlichen Stoffen1 (Chemikaliengesetz - ChemG). https://www. gesetze-im-internet.de/chemg/BJNR017180980. html. Zugegriffen: 19. Okt. 2021

14. Bundesanstalt für Arbeitsschutz und Arbeitsmedizin Allgemeinverfügung Az: 5.0-710 30/01.00003, Fassung vom 15. April 2020.https://www.baua.de/ DE/Angebote/Aktuelles/Meldungen/2020/2020-
09-16-Haendedesinfektion.html.Zugegriffen: 19 Okt. 2021

15. Bundesanstalt für Arbeitsschutz und Arbeitsmedizin (2020) Allgemeinverfügung zur Zulassung Ethanol-haltiger, Chloramin-T-haltiger und $\mathrm{Na}$ triumhypochlorit-haltiger Biozidprodukte zur Flächendesinfektion zur Abgabe an und Verwendung durch berufsmäßige Verwender aufgrund einer Gefahr für die öffentliche Gesundheit (Fassung vom 2. April 2020)

16. Bundesinstitut für Arzneimittel und Medizinprodukte (BfArM) (2020) Allgemeinverfügung zu zeitlich befristeten Abweichungen vom Inhalt der Zulassung von alkoholhaltigen Arzneimitteln zur Händedesinfektion. https:// www.bfarm.de/SharedDocs/Downloads/DE/ Service/Presse/Corona/Allgemeinverfuegung_ Haendedesinfektion.pdf. Zugegriffen: 19. Okt. 2021

17. WHO (2009) Guide to local production: WHOrecommended Handrub formulations. www.who. int/gpsc/5may/Guide_to_Local_Production.pdf. Zugegriffen: 19.0kt. 2021

18. Suchomel M, Kundi M, PittetD, Weinlich M, RotterM (2012) Testing of the World Health Organization recommended formulations in their application as hygienic hand rubs and proposals for increased efficacy. Am J Infect Control 40(4):328-331

19. Siddharta A, Pfaender S, Vielle NJ et al (2017) Virucidal activity of world health organizationrecommended formulations against enveloped viruses, including Zika, Ebola, and emerging Coronaviruses. J Infect Dis 215(6):902-906

20. Steinmann J, Becker B, Bischoff B et al (2010) Virucidal activity of 2 alcohol-based formulations proposed as hand rubs by the World Health Organization. Am J Infect Control 38(1):66-68

21. Kratzel A, Todt D, V'kovski Pet al (2020) Inactivation of severeacute respiratorysyndrome Coronavirus 2 by WHO-recommended hand rub formulations and alcohols. Emerg Infect Dis. https://doi.org/10. 3201/eid2607.200915

22. Bundesinstitut für Arzneimittel und Medizinprodukte (BfArM) (2004) Anlage zur zehnten Verordnung zur Änderung der Verordnung über Standardzulassungen von Arzneimitteln vom 6 Dezember 2004, Anlageband zum Bundesgesetzblatt Teil INr.67 vom 15. Dezember 2004

23. Bundesinstitut für Arzneimittel und Medizinprodukte (BfArM) (2006) Elfte Verordnung zur Änderung der Verordnung über Standardzulassungen von Arzneimitteln vom 19. Oktober 2006. Bundesgesetzblatt 2006 Teil I Nr. 48, vom 26. Oktober 2006

24. Liste der vom Robert Koch-Institut geprüften und anerkannten Desinfektionsmittel und -verfahren, Stand: 31. Oktober 2017 (17. Ausgabe). https://www.rki. de/DE/Content/Infekt/Krankenhaushygiene/ Desinfektionsmittel/Downloads/BGBI_60_2017_ Desinfektionsmittelliste.pdf. Zugegriffen: 19 . Okt. 2021

25. Wirkbereich „begrenzt viruzid“ für Isopropanol 70 Vol.\% zur hygienischen Händedesinfektion, Mitteilung vom 12.3.2020. https://www.rki. de/DE/Content/Infekt/Krankenhaushygiene/ Desinfektionsmittel/Desinfektionsmittellist/ Isopropanol.html.Zugegriffen: 19.0kt. 2021

26. Qualitätskennzeichen für den Einkauf von Händedesinfektionsmitteln. Mitteilung der Desinfektionsmittel-Kommission im VAH zum internationalen Tag der Händehygiene am 05.05.2020. https:// vah-online.de/files/download/vah-mitteilungen/ 
73_75_VAH_Einkauf\%20HDM_HM_5_20.pdf. Zugegriffen: 19.0kt. 2021

27. Kampf G, Exner M, Schwebke I, Gebel J (2021) Ethanol is indispensable and safe as a biocidal active substance for hand disinfection. J Hosp Infect 108:205-206. https://doi.org/10.1016/j. jhin.2020.11.013

28. https://www.gov.bm/sites/default/files/ Methanol-Contaminated-Hand-SanitizersRecall-List.pdf.Zugegriffen: 19.0kt. 2021

29. Dear K, Grayson L, Nixon R (2020) Potential methanol toxicity and the importance of using a standardised alcohol-based hand rub formulation in the era of COVID-19. Antimicrob Resist Infect Control 9(1):129. https://doi.org/10.1186/s13756020-00788-5

30. Produktwarnung EDEKA. https://www. produktwarnung.eu/2020/11/06/rueckrufvon-desinfektions-handgel-via-edeka-undmarktkauf/21329.Zugegriffen: 19. Okt. 2021

31. Verordnung über die Meldung von Biozid-Produkten nach dem Chemikaliengesetz (Biozid-Meldeverordnung-ChemBiozidMeldeV). https://www. gesetze-im-internet.de/chembiozidmeldev 2011/BJNR108500011.html. Zugegriffen: 19 Okt. 2021

32. Delegierte Verordnung (EU) Nr. 1062/2014 der Kommission vom 4. August 2014. https:// eur-lex.europa.eu/legal-content/DE/TXT/PDF/? uri=CELEX:32014R1062\&from=LV. Zugegriffen: 19. Okt. 2021

33. Chlorbasierte Desinfektionsmittel: Anforderungen an die Zertifizierung durch den VAH, Mitteilung der Desinfektionsmittel-Kommission im VAH. https://vah-online.de/files/ download/vah-mitteilungen/Chlorbasierte Desinfektionsmittel_107_108_VAH_HM_6_20. pdf.Zugegriffen:19.0kt. 2021

34. Voraussetzungen zur VAH-Zertifizierung chlorbasierter Händedesinfektionsmittel, Mitteilung der Desinfektionsmittel-Kommission im VAH. https:// vah-online.de/files/download/vah-mitteilungen/ VAH_Chlorbasierte\%2OHDM_HM 10_20.pdf.Zugegriffen: 19.0kt. 2021

35. Händehygiene in Einrichtungen des Gesundheitswesens Empfehlung der Kommission für Krankenhaushygiene und Infektionsprävention (KRINKO) beim Robert Koch-Institut (RKI) Bundesgesundheitsbl (2016) 59:1189-1220 DOI https:// doi.org/10.1007/s00103-016-2416-6

36. BAUA Helpdesk 0598. https://www.reachclp-biozid-helpdesk.de/SharedDocs/FAQs/DE/ Biozide/Wirkstoffe/Wirkstoffe-02\%200598.html. Zugegriffen: 19.0kt. 2021

37. Unionsliste der genehmigten Wirkstoffe. https:// www.reach-clp-biozid-helpdesk.de/DE/Biozide/ Wirkstoffe/Genehmigte-Wirkstoffe/GenehmigteWirkstoffe_node.html.Zugegriffen: 19.0kt.2021

38. Kramer A, Schwebke I, Kampf G (2006) How long do nosocomial pathogens persist on animate surfaces. A systematic review. BMCInfect Dis 6:130. https://doi.org/10.1186/1471-2334-6-130

39. Wißmann JE, Kirchhoff $L$, Brüggemann $Y$ et al (2021) Persistence of pathogens on inanimate surfaces: a narrative review. Microorganisms 9:343. https://doi.org/10.3390/microorganisms9020343

40. van Doremalen N, Morris DH, Holbrook MG (2020) Aerosol and surface stability of SARSCoV-2 as compared with SARS-CoV-1. N Engl J Med 382(16):1564-1567.https://doi.org/10.1056/ NEJMc2004973

41. Kratzel A, SteinerS, TodtDetal (2020)Temperaturedepent surface stability of SARS-CoV-2. J Infect
81(3):452-482. https://doi.org/10.1016/j.jinf. 2020.05.074

42. Zhou Y, Zeng Y, Chen C (2020) Presence of SARSCoV-2 RNA in isolation ward environment 28 days after exposure. Int $\mathrm{J}$ Infect Dis 97:258-259. https:// doi.org/10.1016/j.jiji.2020.06.015

43. Zhou J, Otter JA, Price JR et al (2020) Investigating SARS-CoV-2 surface and air contamination in an acute healthcare setting during the peak of the COVID-19 pandemic in London. Clin Infect Dis. https://doi.org/10.1093/cid/ciaa905

44. Ong SWX, Tan YK, Chia PY et al (2020) Air, surface environmental, and personal protective equipment contamination by severe acute respiratory syndrome Coronavirus 2 (SARScoV-2) from a symptomatic patient. JAMA 323(16):1610-1612. https://doi.org/10.1001/ jama.2020.3227

45. Kampf G, Lemmen S, Suchomel M (2020) Ct values and infectivity of SARS-CoV-2 on surfaces. Lancet Infect Dis. https://doi.org/10.1016/S14733099(20)30883-5

46. Kampf G (2020) COVID-19-Infektionsschutz: Ohne Nutzen. Dtsch Arztebl 117(26):A-1332/B-1132

47. (2021) CDC Centers of disease control and prevention Science Brief: SARS-CoV-2 and Surface (Fomite) Transmission for Indoor Community Environments. https://www.cdc.gov/coronavirus/ 2019-ncov/more/science-and-research/surfacetransmission.html.Zugegriffen: 19.0kt. 2021

48. Santarpia JL, Rivera DN, Herrera VL et al (2020) Aerosol and surface contamination of SARS-CoV-2 observed in quarantine and isolation care. Sci Rep 10:13892

49. Eggers M, Schwebke I, Suchomel M et al (2021) The European tiered approach for virucidal efficacy testing - rationale for rapidly selecting disinfectants against emerging and re-emerging viral diseases. Euro Surveill. https://doi. org/10.2807/1560-7917.ES.2021.26.3.2000708. (https://www.eurosurveillance.org/content/10. 2807/1560-7917.ES.2021.26.3.2000708.Zugegriffen 19. Okt. 2021)

50. Steinhauer K, Meister TL, Todt D et al (2021) Virucidal efficacy of different formulations for hand and surface disinfection targeting SARS CoV-2. J Hosp Infect 112:27-30. https://doi.org/10.1016/ j.jhin.2021.03.015 